\title{
Massive Computation for Understanding Core-Collapse Supernova Explosions
}

\author{
Christian D. Ott ${ }^{1,2}$ \\ ${ }^{1}$ TAPIR, Walter Burke Institute for Theoretical Physics, California Institute of Technology, Pasadena, CA, 91125, USA \\ ${ }^{2}$ Yukawa Institute for Theoretical Physics, Kyoto University, Kyoto, Japan \\ How do massive stars explode? Progress toward the answer is driven by increases in compute power. Petascale supercomputers \\ are enabling detailed three-dimensional simulations of core-collapse supernovae. These are elucidating the role of fluid instabilities, \\ turbulence, and magnetic field amplification in supernova engines.
}

Index Terms-Supernovae, neutron stars, gravitational collapse

\section{INTRODUCTION}

Core-collapse supernova explosions come from stars more massive than $\sim 8-10$ times the mass of the Sun. Ten core-collapse supernovae explode per second in the universe, automated astronomical surveys discover multiple per night, and one or two explode per century in the Milky Way. Corecollapse supernovae outshine entire galaxies in photons for weeks and output more power in neutrinos than the combined light output of all other stars in the universe, for tens of seconds. These explosions pollute the interstellar medium with the ashes of thermonuclear fusion. From these elements, planets form and life is made. Supernova shock waves stir the interstellar gas, trigger or shut off the formation of new stars, and eject hot gas from galaxies. At their centers, a strongly gravitating compact remnant, a neutron star or a black hole, is formed.

As the name alludes, the explosion is preceded by collapse of a stellar core. At the end of its life, a massive star has a core composed mostly of iron-group nuclei. The core is surrounded by an onion-skin structure of shells dominated by successively lighter elements. Nuclear fusion is still ongoing in the shells, but the iron core is inert. The electrons in the core are relativistic and degenerate. They provide the lion's share of the pressure support stabilizing the core against gravitational collapse. In this, the iron core is very similar to a white dwarf star, the end product of low-mass stellar evolution. Once the iron core exceeds its maximum mass (the so-called effective Chandrasekhar mass of $\sim 1.5-2$ solar masses $\left[M_{\odot}\right]$ ), gravitational instability sets in. Within a few tenths of a second, the inner core collapses from a central density of $\sim 10^{10} \mathrm{~g} \mathrm{~cm}^{-3}$ to a density comparable to that in an atomic nucleus $\left(\gtrsim 2.7 \times 10^{14} \mathrm{~g} \mathrm{~cm}^{-3}\right)$. There, the repulsive part of the nuclear force causes a stiffening of the equation of state (EOS; the pressure-density relationship). The inner core first overshoots nuclear density, then rebounds ("bounces") into the still collapsing outer core. The inner core then stabilizes and forms the inner regions of the newborn protoneutron star. The hydrodynamic supernova shock is created at the interface of inner and outer core. First, the shock moves outward dynamically. It then quickly loses energy by work

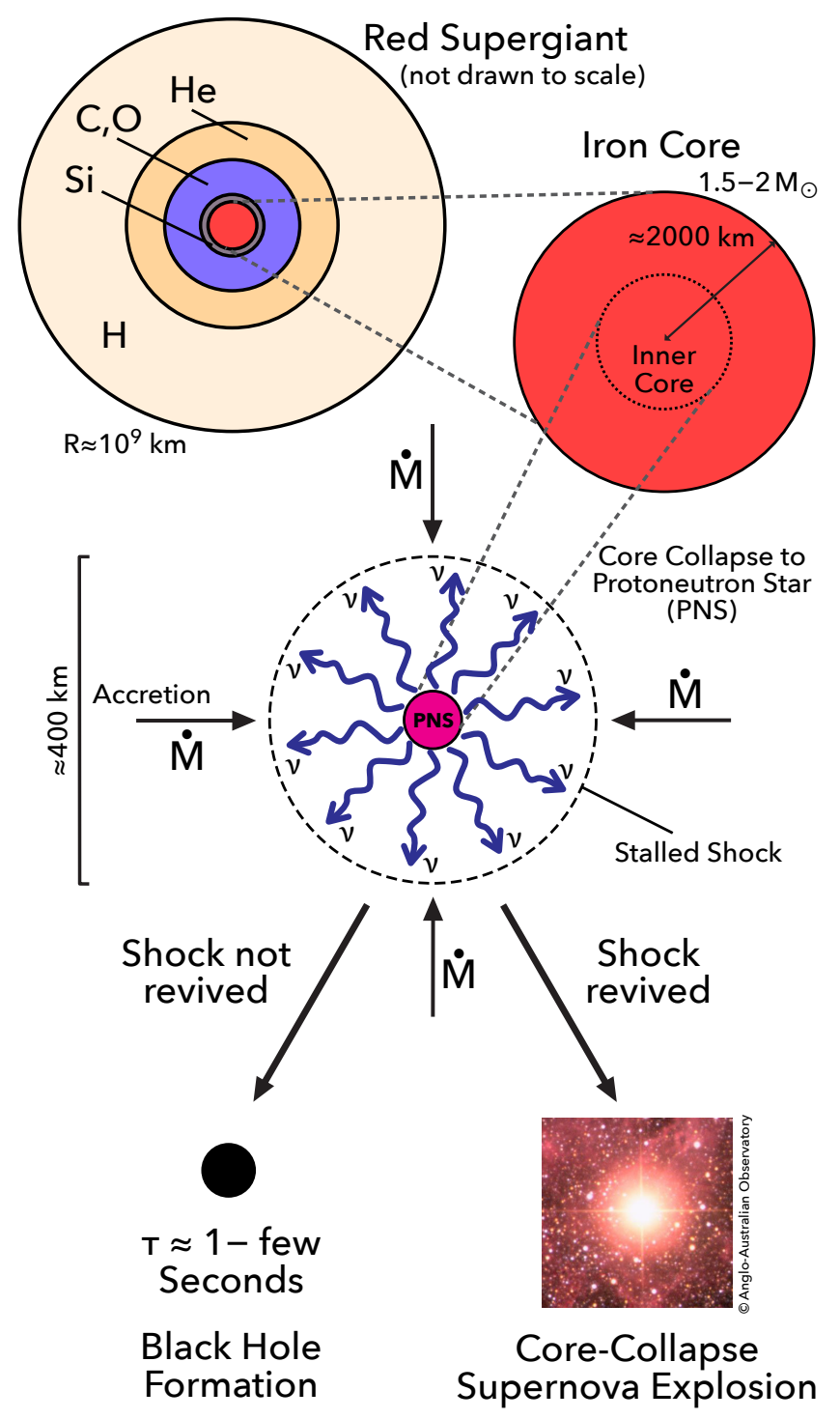

Fig. 1. Schematic of core collapse and its simplest outcomes. The image shows SN 1987A, which exploded in the Large Magellanic Cloud. 
done breaking up infalling iron-group nuclei into neutrons, protons, and alpha particles. The copious emission of neutrinos from the hot $\left(T \sim 10 \mathrm{MeV} \simeq 10^{11} \mathrm{~K}\right)$ gas further reduces energy and pressure behind the shock. The shock stalls and turns into an accretion shock: the ram pressure of accretion of the star's outer core balances the pressure behind the shock.

The supernova mechanism must revive the stalled shock to drive a successful core-collapse supernova explosion. Depending on the structure of the progenitor star, this must occur within one to a few seconds of core bounce. Otherwise, continuing accretion pushes the protoneutron star over its maximum mass $\left(\sim 2-3 M_{\odot}\right)$, which results in the formation of a black hole and no supernova explosion.

If the shock is successfully revived, it must travel through the outer core and the stellar envelope before it breaks out of the star and creates the spectacular explosive display observed by astronomers on Earth. This may take more than a day for a red supergiant star (e.g., like Betelgeuse, a $\sim 20 M_{\odot}$ star in the constellation Orion) or just tens of seconds for a star that has been stripped of its extended hydrogen-rich envelope by a strong stellar wind or mass exchange with a companion star in a binary system.

The photons observed by astronomers are emitted extremely far from the central regions. They carry information on the overall energetics, the explosion geometry, and on the products of explosive nuclear burning that is triggered by the passing shock wave. They can, however, only provide weak constraints on the inner workings of the supernova. Direct observational information on the supernova mechanism can be gained only from neutrinos and gravitational waves that are emitted directly in the supernova core. Detailed computational models are required for gaining theoretical insight and for making predictions that can be contrasted with future neutrino and gravitational-wave observations from the next core-collapse supernova in the Milky Way.

\section{Supernova Energetics And Mechanisms}

Core-collapse supernovae are "gravity bombs." The energy reservoir from which any explosion mechanism must draw is the gravitational energy released in the collapse of the iron core to a neutron star: $\sim 3 \times 10^{53} \mathrm{erg}\left(3 \times 10^{46} \mathrm{~J}\right)$, a mass-energy equivalent of $\sim 0.15 M_{\odot} c^{2}$. A fraction of this tremendous energy is stored initially as heat (and rotational kinetic energy) in the protoneutron star and the rest comes from its subsequent contraction. Astronomical observations, on the other hand, show the typical core-collapse supernova explosion energy to be in the range $10^{50}-10^{51} \mathrm{erg}$. Hypernova explosions may have up to $10^{52} \mathrm{erg}$, but they make up $\lesssim 1 \%$ of all core-collapse supernovae. A small subset of hypernovae are associated with gamma-ray bursts.

Where is all the gravitational energy going that does not contribute to the explosion energy? The answer is: Neutrinos. Antineutrinos and neutrinos of all flavors carry away $\gtrsim 99 \%$ ( $\gtrsim 90 \%$ in the hypernova case) of the available energy over $\mathcal{O}(10) \mathrm{s}$ as the protoneutron star cools and contracts. This was first theorized and then later observationally confirmed with the detection of neutrinos from SN 1987A, the most recent core-collapse supernova in the Milky Way vicinity.

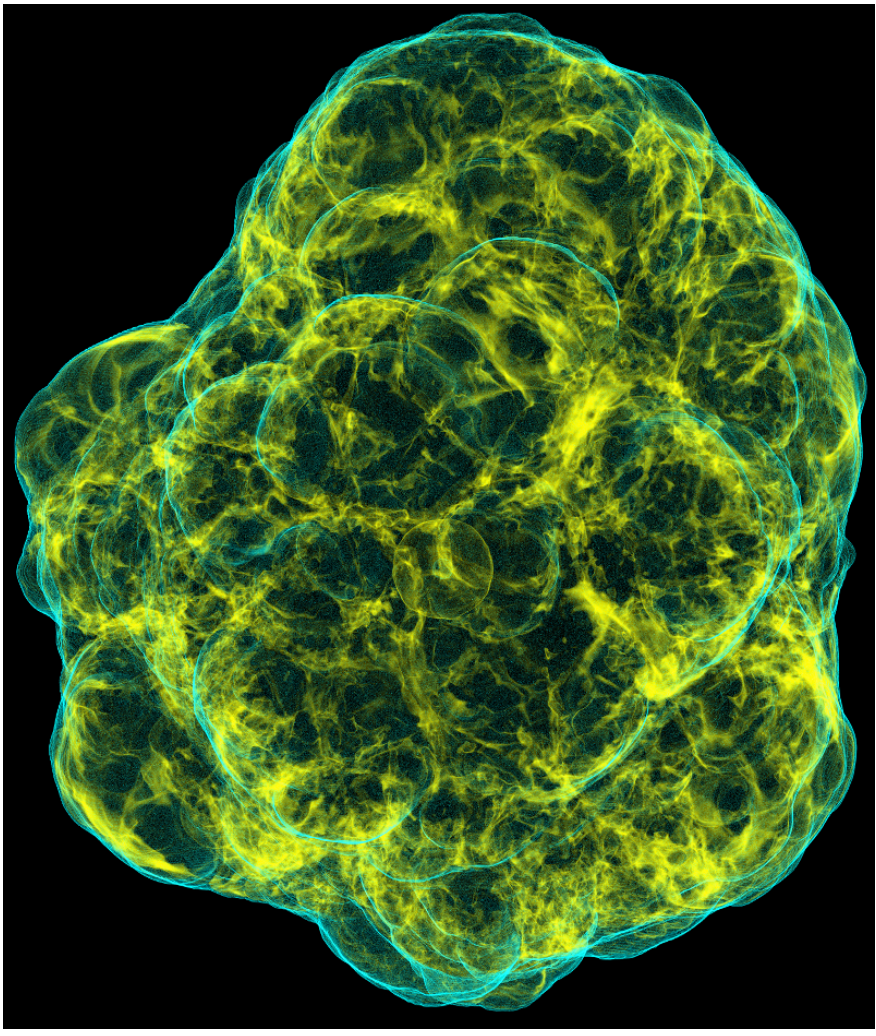

Fig. 2. Volume rendering of the specific entropy in the core of a neutrinodriven core-collapse supernova at the onset of explosion. Based on the 3D general-relativistic simulations of [1] and rendered by Steve Drasco (Cal Poly San Luis Obispo). Specific entropy is a preferred quantity for visualization, since in the core of a supernova, it typically ranges from $\sim 1$ to $\sim 20$ units of Boltzmann's constant $k_{\mathrm{B}}$ per baryon. Shown is the large-scale asymmetric shock front and a layer of hot expanding plumes behind it. The physical scale is roughly $600 \times 400 \mathrm{~km}$.

Since neutrinos dominate the energy transport through the supernova, they might quite naturally have something to do with the explosion mechanism. The neutrino mechanism, in its current form, was proposed by Bethe \& Wilson [2]. In this mechanism, a fraction $(\sim 5 \%)$ of the outgoing electron neutrinos and antineutrinos is absorbed in a layer between protoneutron star and the stalled shock. In the simplest picture, this neutrino heating increases the thermal pressure behind the stalled shock. Consequently, the dynamical pressure balance at the accretion shock is violated and a runaway explosion is launched.

The neutrino mechanism fails in spherical symmetry (1D, e.g., [3]), but is very promising in multiple dimensions (axisymmetry [2D], 3D). This is due largely to multi-D hydrodynamic instabilities that break spherical symmetry (see Figure 2 for an example), increase the neutrino mechanism's efficiency, and facilitate explosion. I will discuss this in more detail later in this article. The neutrino mechanism is presently favored as the mechanism driving most core-collapse supernova explosions (see [3] for a recent review).

Despite its overall promise, the neutrino mechanism is very inefficient. Only $\lesssim 5 \%$ of the outgoing total electron neutrino and antineutrino luminosity is deposited behind the stalled shock at any moment and much of this deposition is lost again 


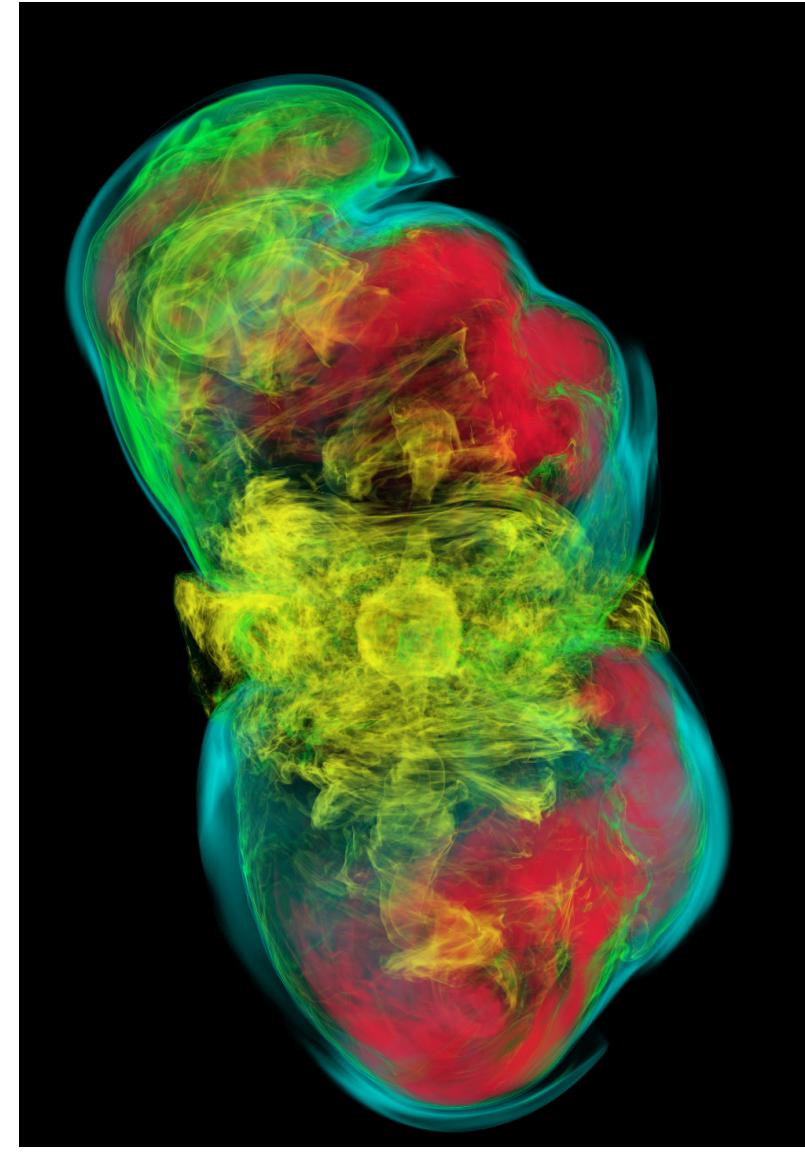

Fig. 3. Volume rendering of the specific entropy in the core of a magnetorotational core-collapse supernova. Bluish colors indicate low entropy, red colors high entropy, and green and yellow intermediate entropy. The vertical is the axis of rotation and shown is a region of $\sim 1600 \times 800 \mathrm{~km}$. The ultrastrong toroidal magnetic field surrounding the the protoneutron star pushes hot plasma out along the rotation axis. The distorted, double-lobe structure is due to an MHD kink instability akin those seen in Tokamak fusion experiments. Used with permission from Mösta et al. 2014 [4].

as heated gas flows down, leaves the heating region, and settles onto the protoneutron. The neutrino mechanism may (barely) be able to power ordinary core-collapse supernovae, but it cannot deliver hypernova explosion energies or account for gamma-ray bursts.

An alternative mechanism that may be part of the explanation for such extreme events is the magnetorotational mechanism, first suggested by Bisnovatyi-Kogan [5] and LeBlanc \& Wilson [6]. In its modern form, a very rapidly spinning core collapses to a protoneutron star with a spin period of only $\sim 1$ millisecond. Its core is expected to be spinning uniformly, but its outer regions will be extremely differentially rotating. These are ideal conditions for the magnetorotational instability (MRI, [7]) to operate, amplify any seed magnetic field, and drive magnetohydrodynamic (MHD) turbulence. If a dynamo process is present, an ultra-strong large-scale (globally ordered) magnetic field is built up. This makes the protoneutron star a protomagnetar. Provided this occurs, magnetic pressure gradients and hoop stresses could lead to outflows along the axis of rotation. The MRI's fastest growing mode has a small wavelength and is extremely difficult to resolve numerically.
Because of this, all simulations of the magnetorotational mechanism to date have simply made the assumption that a combination of MRI and dynamo is operating. They then adhoc imposed a strong large-scale field as an initial condition. In 2D simulations, collimated jets develop along the axis of rotation. In $3 \mathrm{D}$, the jets are unstable and a more complicated explosion geometry develops [4], as shown in Figure 3. Nevertheless, even in $3 \mathrm{D}$, an energetic explosion could potentially be powered.

The magnetorotational mechanism requires one special property of the progenitor star: rapid core rotation. Presently, stellar evolution theory suggests that the cores of most massive stars should be slowly spinning. However, there may be exceptions of rapidly spinning cores at just about the right occurrence rate to explain hypernovae and long gamma-ray bursts.

Besides the neutrino mechanism and the magnetorotational mechanism, a number of other explosion mechanisms have been proposed. I direct the interested reader to the more extensive review by [3].

\section{A Multi-Scale, Multi-Physics, Multi-Dimensional Computational Challenge}

The core-collapse supernova problem is highly complex, inherently non-linear, and involves many branches of (astro)physics. Only limited progress can be made with analytic or perturbative methods. Computational simulation is a powerful means for gaining theoretical insight and for making predictions that could be tested with astronomical observations of neutrinos, gravitational waves, and electromagnetic radiation.

Core-collapse supernova simulations are time evolution simulations - starting from initial conditions, the matter, radiation, and gravitational fields are evolved in time. In the case of time-explicit evolution, the numerical timestep is limited by causality, controlled by the speed of sound in Newtonian simulations, and the speed of light in general-relativistic simulations. Because of this, an increase in the spatial resolution by a factor of two corresponds to a decrease in the time step by a factor of two. Hence, in a 3D simulation, the computational cost scales with the fourth power of resolution.

\section{A. Multi Scale}

Taking the red supergiant in Figure 1 as an example, a complete core-collapse supernova simulation that follows the shock to the stellar surface, would have to cover dynamics on a physical scale from $\sim 10^{9} \mathrm{~km}$ (stellar radius) down to $\sim 0.1 \mathrm{~km}$ (the typical scale over which structure and thermodynamics of the protoneutron star change). These ten orders of magnitude in spatial scale are daunting. In practice, reviving the shock and tracking its propagation to the surface can be treated as (almost) independent problems. If our interest is on the shock revival mechanism, we need to include the inner $\sim 10,000 \mathrm{~km}$ of the star. Since information about core collapse is communicated to overlying layers with the speed of sound, stellar material at greater radii will not "know" that core collapse has occurred before it is hit by the revived expanding shock. 
Even with only five decades in spatial scale, some form of grid refinement or adaptivity is called for: a 3D finitedifference grid with an extent of $10,000 \mathrm{~km}$ symmetric about the origin with uniform $0.1 \mathrm{~km}$ cell size would require 57 PB of RAM to store a single double precision variable. Many tens to hundreds of 3D variables are required. Such high uniform resolution is not only currently impossible but also unnecessary. Most of the resolution is needed near the protoneutron star and in the region behind the stalled shock. The near-free-fall collapse of the outer core can be simulated with much lower resolution.

Because of the broad range of physics involved (see below) and the limited available compute power, early core-collapse supernova simulations were spherically symmetric (1D). 1D simulations often employ a Lagrangian, comoving mass coordinate discretization. This grid can be set up to provide just the right resolution where and when needed or can be dynamically re-zoned (an adaptive mesh refinement [AMR] technique). Other 1D codes discretize in the Eulerian frame and use a fixed grid whose cells are radially stretched using geometric progression.

In 2D simulations, Eulerian, geometrically-spaced fixed spherical grids are the norm, but some codes use cylindrical coordinates and AMR. Spherical grids, already in 2D, suffer from a coordinate singularity at the axis that can lead to numerical artifacts. In 3D, they become even more difficult to handle and their focusing grid lines impose a severe timestep constraint near the origin. Some 3D codes still use a spherical grid, while many others employ Cartesian AMR grids. Recent innovative approaches use so-called multi-block grids with multiple curvilinear touching or overlapping logically Cartesian "cubed-sphere" grids (e.g., [8]).

\section{B. Multi Physics}

Core-collapse supernovae are very rich in physics. All fundamental forces are involved and essential to the core collapse phenomenon. These forces are probed under conditions that are impossible (or exceedingly difficult) to create in earthbound laboratories.

Gravity drives the collapse and provides the energy reservoir. It is so strong near the protoneutron star that general relativity becomes important and its Newtonian description does not suffice. The electromagnetic force describes the interaction of the dense, hot magnetized, perfectly conducting plasma and the photons that provide thermal pressure and make the supernova light. The weak force governs the interactions of neutrinos and the strong (nuclear) force is essential in the nuclear EOS and nuclear reactions.

All this physics occurs at the microscopic, per particle level. Fortunately, the continuum assumption holds, allowing us to describe core-collapse supernovae on a macroscopic scale by a coupled set of systems of non-linear partial differential equations (PDEs):

- (Magneto)hydrodynamics (MHD). The stellar plasma is in local thermodynamic equilibrium, essentially perfectly conducting, and essentially inviscid (though neutrinos may provide some shear viscosity in the protoneutron

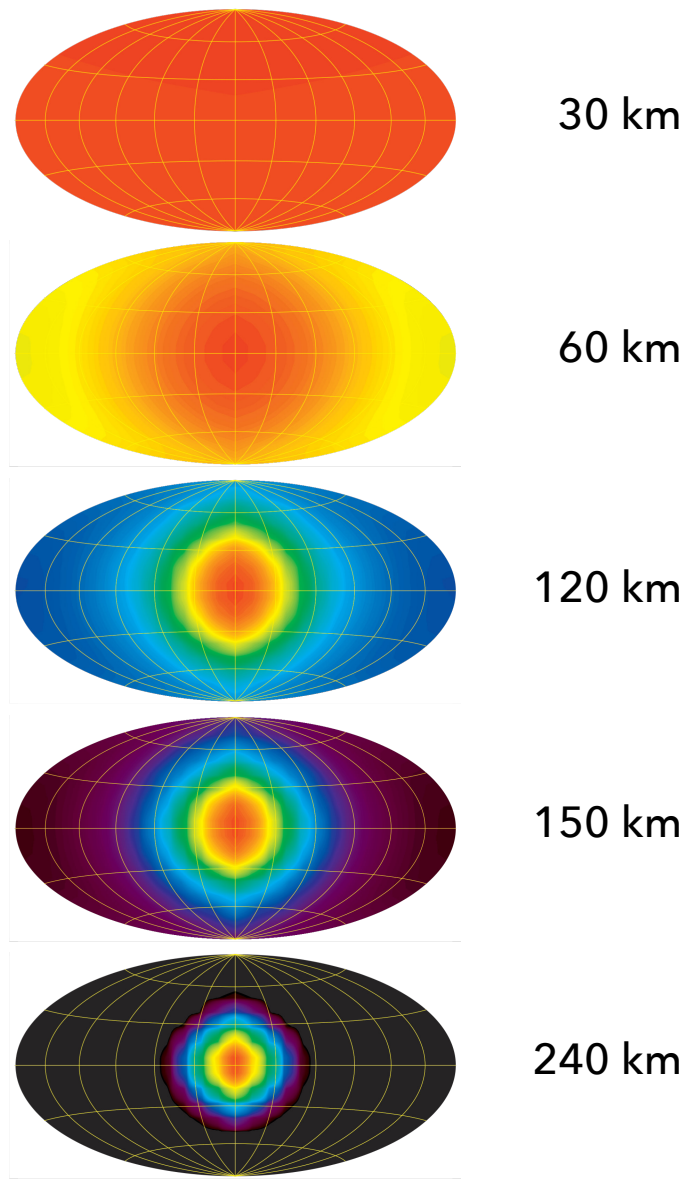

Fig. 4. Map projections of the momentum-space neutrino radiation field (for $\nu_{e}$ at an energy of $16.3 \mathrm{MeV}$ ) going outward radially (from top to bottom) on the equator of a supernova core. Generated using the simulation results of $[9 \mid$. Inside the protoneutron $\operatorname{star}(R \lesssim 30 \mathrm{~km})$ neutrinos and matter are in equilibrium and the radiation field is isotropic. It becomes more and more forward peaked as the neutrinos decouple and become free streaming. Handling the transition from slow diffusion to free streaming correctly requires angle-dependent radiation transport, which is a $6+1 \mathrm{D}$ problem and computationally extremely challenging.

star). The ideal, inviscid MHD approximation is appropriate under these conditions. The MHD equations are hyperbolic and can be written in flux-conservative form with source terms that do not include derivatives of the MHD variables. They are typically solved with standard time-explicit high-resolution shock capturing methods that exploit the characteristic structure of the equations (e.g., [10]). Special attention must be paid to preserving the divergence-free property of the magnetic field. The MHD equations require an EOS as a closure (see below).

Unless ultra-strong $\left(B \gtrsim 10^{15} \mathrm{G}\right)$, magnetic fields have little effect on the supernova dynamics and thus are frequently neglected. Since strong gravity and velocities up to a few tenths of the speed of light are involved, the MHD equations are best solved in a general-relativistic formulation. General-relativistic MHD is computationally particularly expensive, because the conserved variables are not the primitive variables (density, internal energy / temperature, velocity, chemical composition). The latter are needed for the EOS and enter flux terms. After 
each update, they must be recovered from the conserved variables via multi-dimensional root finding.

- Gravity. Deviations in the strength of the gravitational acceleration between Newtonian and general-relativistic gravity are small in the precollapse core, but become of order $10-20 \%$ in the protoneutron star phase. In the case of black hole formation, Newtonian physics breaks down completely. General relativistic gravity is included at varying levels in simulations. Some neglect it completely and solve the linear elliptic Newtonian Poisson equation to compute the gravitational potential. This is done using direct multigrid methods or integral multipole expansion methods. Some codes modify the monopole term in the latter approach to approximate general relativistic effects.

Including full general relativity is more challenging, in particular in $2 \mathrm{D}$ and $3 \mathrm{D}$, since there general relativity has radiative degrees of freedom (gravitational waves). An entire subfield of gravitational physics, numerical relativity, spent nearly five decades looking for ways to solve Einstein's equations on computers (see [11] for a comprehensive introduction). In general relativity, changes in the gravitational field propagate at the speed of light. Hence, time evolution equations must be solved. This is done by splitting 4D spacetime into 3D spatial slices that are evolved in the time direction. In the simplest way of writing the equations (the so-called Arnowitt-Deser-Misner [ADM] formulation), they form a system of 12 partial differential evolution equations, 4 gauge variables that must be specified (and evolved in time or recalculated on each slice), and 4 elliptic constraint equations without time derivatives. The ADM formulation has poor numerical stability properties. These lead to violations of the constraint equations and numerical instabilities that make long-term evolution impossible.

It took until the 2000s for numerical relativity to find formulations of Einstein's equations and gauge choices that together lead to stable long-term evolutions. In some cases, well-posedness and strong or symmetric hyperbolicity can be proven. The equations are typically evolved time-explicitly with straightforward high-order (fourth and higher) finite difference schemes or with multi-domain pseudospectral methods.

Since numerical relativity only recently became applicable to astrophysical simulations, very few core-collapse supernova codes are fully general relativistic at this point [1], [12]. The fully general-relativistic approach is much more memory and FLOP intensive than solving the Newtonian Poisson equation. Its advantage in largescale computations, however, is the hyperbolic nature of the equations, which does not require global matrix inversions or summations and thus is advantageous for the parallel scaling of the algorithm.

- Neutrino Transport and Neutrino-Matter Interactions. Neutrinos move at the speed of light (the very small neutrino masses are neglected) and can travel macroscopic distances between interactions. Therefore, they must be treated as non-equilibrium radiation. Radiation transport is closely related to kinetic theory's Boltzmann equation. It describes the phase-space evolution of the neutrino distribution function or, in radiation transport terminology, their specific intensity. This is a $6+1 \mathrm{D}$ problem: 3 spatial dimensions, neutrino energy, and two momentum space propagation angles in addition to time. The angles describe the directions from which neutrinos are coming and where they are going at a given spatial coordinate. In addition, the transport equation must be solved separately for multiple neutrino species: electron neutrinos, electron antineutrinos, and heavy-lepton $(\mu, \tau)$ neutrinos and antineutrinos.

Figure 4 shows map projections of the momentum space angular neutrino distribution at different radii in a supernova core. In the dense protoneutron star, neutrinos are trapped and in equilibrium with matter. Their radiation field is isotropic. They gradually diffuse out and decouple from matter at the neutrinosphere (the neutrino equivalent of the photosphere). This decoupling is gradual and marked by the transition of the angular distribution into the forward (radial) direction. In the outer decoupling region, neutrino heating is expected to occur and the heating rates are sensitive to the angular distribution of the radiation field (cf. [9]). Eventually, at radii of a few hundred kilometers, the neutrinos have fully decoupled and are free streaming. Neutrino interactions with matter (and thus the decoupling process) are very sensitive to neutrino energy, since weak-interaction crosssections scale with the square of the neutrino energy. This is why neutrino transport needs to be multi-group, with typically a minimum of $10-20$ energy groups covering supernova neutrino energies of $1-\mathcal{O}(100) \mathrm{MeV}$. Typical mean energies of electron neutrinos are around $10-30 \mathrm{MeV}$. Energy exchanges between matter and radiation occur via the collision terms in the Boltzmann equation. These are stiff sources/sinks that must be handled time-implicitly with (local) backward-Euler methods. The neutrino energy bins are coupled through (1) frame-dependent energy shifts since the material neutrinos interact with is moving, (2) gravitational redshift, and (3) energy transfer in scatterings off of electrons and nucleons. Neutrino-matter interaction rates are usually precomputed and stored in dense multi-D tables within which simulations interpolate.

Full 6+1 D general-relativistic Boltzmann neutrinoradiation hydrodynamics is exceedingly challenging and has so far not been possible to included in core-collapse supernova simulations. $3+1 \mathrm{D}$ (1D in space, 2D in momentum space) (e.g., [13]), 5+1 D (2D in space, 3D in momentum space) simulations [9] and static 6D simulations [14] have been carried out.

Most (spatially) multi-D simulations treat neutrino transport in some dimensionally-reduced approximation. The most common is an expansion of the radiation field into angular moments. The $n$-th moment of this expansion requires information about the $(n+1)$-th moment (and in some cases also about the $(n+2)$-th moment). This necessitates a closure relation for the moment at which 
the expansion is truncated. Multi-group flux-limited diffusion evolves the 0 -th moment (the radiation energy density). The flux limiter is the closure that interpolates between diffusion and free streaming. The disadvantages of this method are its very diffusive nature that washes out spatial variations of the radiation field, its sensitivity to the choice of flux limiter, and the need for time-implicit integration (involving global matrix inversion) due to the stability properties of the parabolic diffusion equation. Two-moment transport is the next better approximation. It solves equations for the radiation energy density and momentum (i.e. the radiative flux) and requires a closure that describes the radiation pressure tensor (also known as the Eddington tensor). This closure can be analytic and based on the local values of energy density and flux (the M1 approximation). Alternatively, some codes compute a global closure based on the solution of a simplified, timeindependent Boltzmann equation. The major advantage of the two-moment approximation is that its advection terms are hyperbolic and can be handled with standard time-explicit finite-volume methods of computational hydrodynamics and only the local collision terms need timeimplicit updates.

There are now implementations of multi-group twomoment neutrino radiation-hydrodynamics in multiple 2D/3D core-collapse supernova simulation codes (e.g., [12], [15], [16]). This method may be sufficiently close to the full Boltzmann solution (in particular if a global closure is used) and appears to be the way toward massively-parallel long-term 3D core-collapse supernova simulations.

- Neutrino Oscillations. Neutrinos have mass and can oscillate between flavors. The oscillations occur in vacuum, but can also be mediated by neutrino-electron scattering (the Mikheyev-Smirnov-Wolfenstein [MSW] effect) and neutrino-neutrino scattering. Neutrino oscillations depend on neutrino mixing parameters and on the neutrino mass eigenstates (the magnitudes of the mass differences are known, but not their signs). Observation of neutrinos from the next galactic core-collapse supernova could help constrain the neutrino mass hierarchy (see the recent review by [17]).

MSW oscillations occur in the stellar envelope. They are important for the neutrino signal observed in detectors on Earth, but they cannot influence the explosion itself. The self-induced (via neutrino-neutrino scattering) oscillations, however, occur at the extreme neutrino densities near the core. They offer a rich phenomenology that includes collective oscillation behavior of neutrinos (see the review in [17]). The jury is still out on their potential influence on the explosion mechanism.

Collective neutrino oscillation calculations (essentially solving coupled Schrödinger-like equations) are computationally intensive [17]. They are currently performed independently of core-collapse supernova simulations and do not take into account feedback on the stellar plasma. Fully understanding collective oscillations and their im- pact on the supernova mechanism will quite likely require that neutrino oscillations, transport, and neutrino-matter interactions are solved for together in a quantum-kinetic approach [18].

- Equation of State and Nuclear Reactions. The EOS is essential for the (M)HD part of the problem and for updating the matter thermodynamics after neutrinomatter interactions. Baryons (proton, neutrons, alpha particles, heavy nuclei), electrons, positrons, and photons contribute to the EOS. Neutrino momentum transfer contributes an effective pressure that is taken into account separately since neutrinos are not everywhere in local thermodynamic equilibrium with the stellar plasma. In different parts of the star, different EOS physics applies.

At low densities and temperatures below $\sim 0.5 \mathrm{MeV}$ $\left(\sim 5 \times 10^{9} \mathrm{~K}\right)$, nuclear reactions are too slow to reach nuclear statistical equilibrium. In this regime, the mass fractions of the various heavy nuclei (isotopes, in the following) must be tracked explicitly. As the core collapses, the gas heats up and nuclear burning must be tracked with a nuclear reaction network, a stiff system of ODEs. Solving the reaction network requires the inversion of sparse matrices at each grid point. Depending on the number of isotopes tracked (ranging, typically from $\mathcal{O}(10)$ to $\mathcal{O}(100)$ ), nuclear burning can be a significant contributor to the overall computational cost of a simulation. The EOS in the burning regime is simple, since all isotopes can essentially be treated as non-interacting ideal Boltzmann gases. Often, corrections for Coulomb interactions are included. Photons and electrons/positrons can be treated everywhere as ideal Bose and Fermi gases, respectively. Since electrons will be partially or completely degenerate, computing the electron/positron EOS involves the FLOP-intensive solution of Fermi integrals. Because of this, their EOS is often included in tabulated form.

At temperatures above $\sim 0.5 \mathrm{MeV}$, nuclear statistical equilibrium holds. This greatly simplifies things, since now the electron fraction $Y_{e}$ (number of electrons per baryon; because of macroscopic charge neutrality, $Y_{e}$ is equal to $Y_{p}$, the number fraction of protons) is the only compositional variable. The mass fractions of all other baryonic species can be obtained by solving Sahalike equations for compositional equilibrium. At densities below $\sim 10^{10}-10^{11} \mathrm{~g} \mathrm{~cm}^{-3}$ the baryons can still be treated as ideal Boltzmann gases (but including Coulomb corrections).

The nuclear force becomes relevant at densities near and above $10^{10}-10^{11} \mathrm{~g} \mathrm{~cm}^{-3}$. It is an effective quantum many-body interaction of the strong force and its detailed properties are presently not known. Under supernova conditions, matter will be in NSE in the nuclear regime and the EOS is a function of density, temperature, and $Y_{e}$. Starting from a nuclear force model, an EOS can be obtained in multiple ways (see the [19] for an overview discussion), including direct Hartree-Fock many-body calculations, mean field models, or phenomenological 


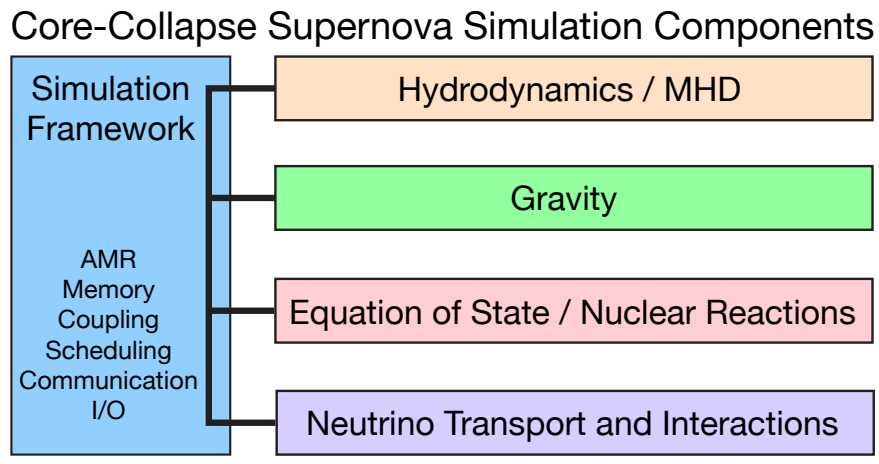

Fig. 5. Multi-physics modules of core-collapse supernova simulation codes. The simulation framework provides parallelization, I/O, execution scheduling, AMR, and memory management.

models (e.g., the liquid-drop model). Typically, the minimum of the Helmholtz free energy is sought and all thermodynamic variables are obtained from derivatives of the free energy. In most cases, EOS calculations are too time consuming to be performed during a simulation. As in the case of the electron/positron EOS, large ( $\gtrsim 200 \mathrm{MB}$; must be stored by each MPI process), densely spaced nuclear EOS tables are precomputed and simulations efficiently interpolate in $\left(\log \rho, \log T, Y_{e}\right)$ to obtain thermodynamic and compositional information.

\section{Effects of Multidimensionality}

Stars are, at zeroth order, gas spheres. It is thus natural to start with assuming spherical symmetry in simulations in particular given the very limited compute power available to the pioneers of supernova simulations. After decades of work, it appears now clear that detailed spherically symmetric simulations robustly fail at producing explosions for stars that are observed to explode in nature. Spherical symmetry itself may be the culprit, since symmetry is clearly broken in corecollapse supernovae:

(i) Observations show that neutron stars receive "birth kicks" giving them typical velocities of $\mathcal{O}(100) \mathrm{km} \mathrm{s}^{-1}$ with respect to the center of mass of their progenitors. The most likely and straightforward explanation for these kicks are highly asymmetric explosions leading to neutron star recoil owing to momentum conservation.

(ii) Deep observations of supernova remnants show that the innermost supernova ejecta exhibit low-mode asphericity similar to the geometry of the shock front shown in Figure 2.

(iii) Analytic considerations and also 1D core-collapse simulations show that the protoneutron star and the region behind the stalled shock where neutrino heating takes place are both unstable to buoyant convection, which always leads to the breaking of spherical symmetry.

(iv) Rotation and magnetic fields naturally break spherical symmetry. Observations of young pulsars show that some neutron stars must be born with rotation periods of order 10 milliseconds. Magnetars may be born with even shorter spin periods if their magnetic field is derived from rapid differential rotation. (v) Multi-D simulations of the violent nuclear burning in the shells overlying the iron core show that large-scale deviations from sphericity develop that couple into the precollapse iron core via the excitation of non-radial pulsations [20]. These create perturbations from which convection will grow after core bounce.

Given the above, multi-D simulations are essential for studying the dynamics of the supernova engine.

The rapid increase of compute power since the early 1990s has facilitated increasingly detailed 2D radiationhydrodynamics simulations over the past two and a half decades. 3D simulations with simplified neutrino treatments have been carried out since the early 2000s. The first 3D neutrino radiation-hydrodynamics simulations have become possible only in the past few years, thanks to the compute power of large petascale systems like US NSF/NCSA Blue Waters, US DOE/ORNL Titan or the Japanese K computer.

\section{Core-Collapse Supernova Simulation Codes}

Many 1D codes exist, some are no longer in use, and one is open source and free to download (http://GR1Dcode.org). There are $\sim 10$ (depending on how one counts) multi-D corecollapse supernova simulation codes in the community. Many, in particular the 3D codes, follow the design encapsulated by Figure 5. They employ a simulation framework (e.g., FLASH, http://flash.uchicago.edu/site/flashcode/ or Cactus http://cactuscode.org) that handles domain decomposition, message passing, memory management, AMR, coupling of different physics components, execution scheduling, and I/O.

Given the tremendous memory requirement and FLOPconsumption of the core-collapse supernova problem, these codes are massively parallel and employ both node-local OpenMP and inter-node MP I parallelization. All current codes follow a data-parallel paradigm with monolithic sequential scheduling. This limits scaling, can create load imbalances with AMR, and makes the use of GPU/MIC accelerators challenging, since communication latencies between accelerator and CPU block execution in the current paradigm.

The Caltech Zelmani [1] core collapse simulation package is an example of a 3D core-collapse supernova code. It is based on the open-source Cactus framework, uses 3D AMR Cartesian and multi-block grids, and employs many components provided by the open-source Einstein Toolkit (http: //einsteintoolkit.org). Zelmani has fully general-relativistic gravity and implements general-relativistic MHD. Neutrinos are included either via a rather crude energy-averaged leakage scheme that approximates the overall energetics of neutrino emission and absorption or via a general-relativistic twomoment M1 radiation-transport solver that has recently been deployed on first simulations [16].

In full radiation-hydrodynamics simulations of the corecollapse supernova problem with 8 levels of AMR, Zelmani exhibits good strong scaling with hybrid-OpenMP/MPI to 16,000 cores on NSF/NCSA Blue Waters. At larger core counts, load imbalances due to AMR prolongation and synchronization operations begin to dominate the execution time. 


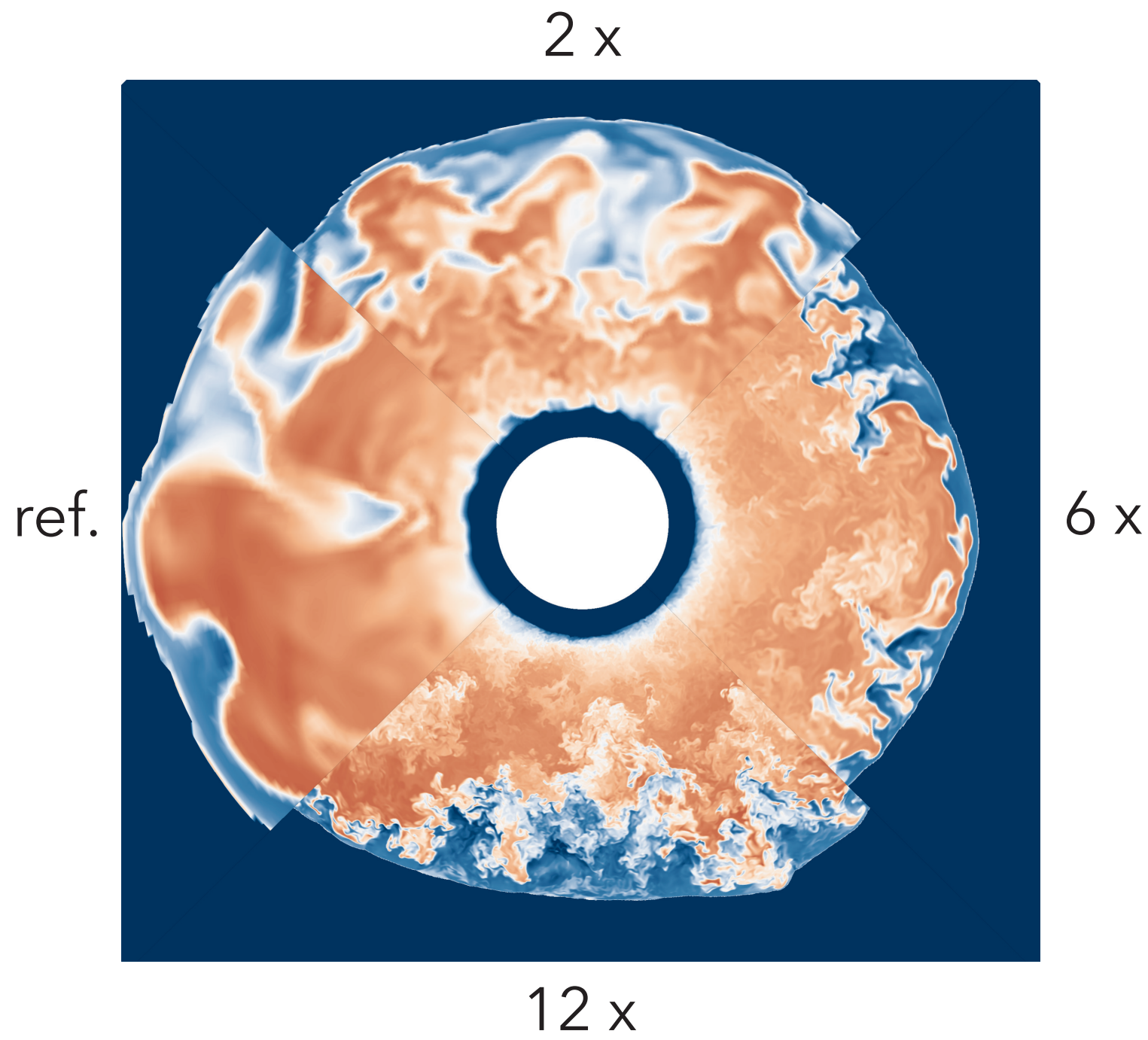

Fig. 7. Slices from four semi-global 3D simulations of neutrino-driven convection with parameterized neutrino cooling and heating, carried out in a $45^{\circ}$ wedge. The colormap is the specific entropy; blue colors mark low entropy region, red colors correspond to high entropy. Only the resolution is varied. The wedge marked "ref." is the reference resolution $\left(\Delta r=3.8 \mathrm{~km}, \Delta \theta=\Delta \varphi=1.8^{\circ}\right)$ that corresponds to the resolution of present global 3D detailed radiation-hydrodynamics core-collapse supernova simulations. Note how low resolution favors large flow features and how the turbulence breaks down to progressively smaller features with increasing resolution. This figure uses simulation results of [21] that includes simulations up to 12 times the reference resolution that were run on 65,536 cores of NSF/NCSA Blue Waters. Rendering by David Radice (Caltech).

\section{Multi-D Dynamics And Turbulence}

Even before the first detailed 2D simulations of neutrinodriven core-collapse supernovae became possible in the mid 1990s, it was clear that buoyant convection in the protoneutron star and in the neutrino-heated region just behind the stalled shock breaks spherical symmetry. Neutrino-driven convection is due to a negative radial gradient in the specific entropy, making the plasma at smaller radii "lighter" than overlying plasma. This is a simple consequence of neutrino heating being strongest at the base of the heating region. Rayleigh-Taylorlike plumes develop from small perturbations and grow to non-linear convection. This convection is extremely turbulent, since the physical viscosity in the heating region is vanishingly small. Neutrino-driven turbulence is anisotropic on large scales (due to buoyancy), mildly compressible (the flow reaches Mach numbers of $\sim 0.5$ ), and only quasi-stationary, because eventually an explosion develops. Nevertheless, it turns out that Kolmogorov's description for isotropic, stationary, incompressible turbulence works surprisingly well for neutrinodriven turbulence (see Figure 6 for a schematic description of Kolmogorov turbulence and [21]).

There is something special about neutrino-driven convection in core-collapse supernovae: unlike convection in globally hydrostatic stars, neutrino-driven convection occurs on top of a downflow of outer core material that has accreted through the stalled shock and is headed for the protoneutron star. The consequence of this is that there is a competition between (i) the time it takes for a small perturbation to grow to macroscopic scale to become buoyant and (ii) the time it takes for it to leave the region that is convectively unstable (the heating region) as it is dragged with the background flow toward the protoneutron star. This means that there are 


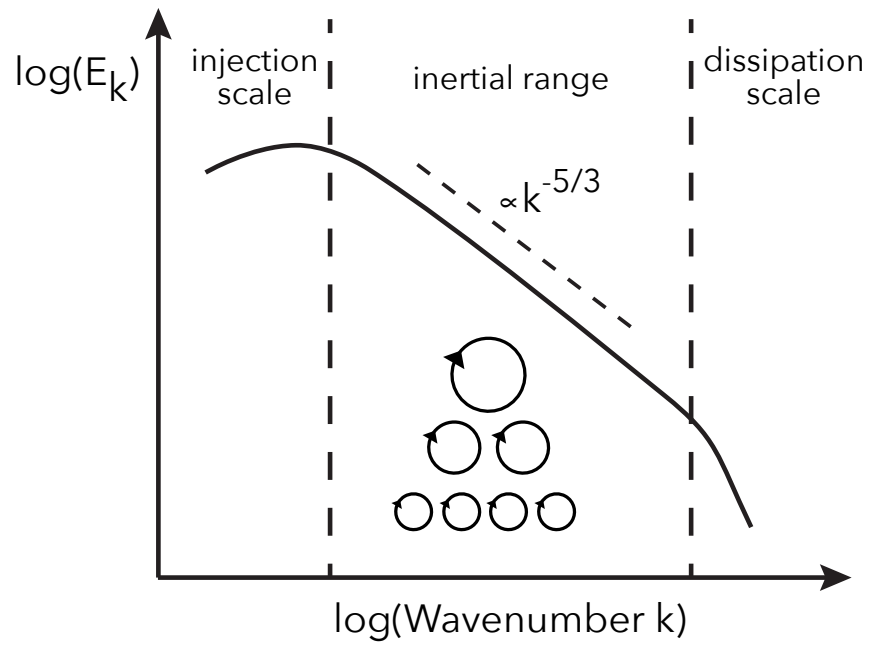

Fig. 6. Schematic view of turbulence: kinetic energy is injected into the flow at large scales and cascades through the inertial range via non-linear interactions of turbulent eddies to small scales (high wavenumbers in the spectral domain) where it dissipates into heat. The scaling of the turbulent kinetic energy with wavenumber in the inertial range is $\propto k^{-5 / 3}$ for Kolmogorov turbulence. This scaling is also found in very high-resolution simulations of neutrinodriven convection 21 .

three parameters governing the appearance of neutrino-driven convection: the strength of neutrino heating, the initial size of perturbations entering through the shock, and the downflow rate through the heating region. Because of this, neutrinodriven convection is not a given and simulations find that it does not develop in some stars.

Even in the absence of neutrino-driven convection, there is another instability that breaks spherical symmetry in the supernova core: the standing accretion shock instability (SASI, [3]). SASI was first discovered in simulations that did not include neutrino heating. It works via a feedback cycle: small perturbations enter through the shock, flow down to the protoneutron star and get reflected as sound waves that in turn perturb the shock. The SASI is a low-mode instability that is most manifest in an up-down sloshing $(\ell=1$ in terms of spherical harmonics) along the symmetry axis in $2 \mathrm{D}$ and in a spiral mode $(m=1)$ in $3 \mathrm{D}$. Once it has reached nonlinear amplitudes, the SASI creates secondary shocks (entropy perturbations) and shear flow from which turbulence develops. SASI appears to dominate in situations in which neutrinodriven convection is weak or absent: in conditions where neutrino heating is weak, the perturbations entering the shock are small, or the downflow rate through the heating region is high.

Independent of how spherical symmetry is broken in the heating region, all simulations agree that $2 \mathrm{D} / 3 \mathrm{D}$ is much more favorable for explosion than 1D. Some 2D and 3D simulations yield explosions for stars where 1D simulations fail (see, e.g., [22]). Why is that?

There are two reasons. The first reason has been known for long and is seemingly trivial: the added degrees of freedom, lateral motion in $2 \mathrm{D}$, and lateral and azimuthal motion in $3 \mathrm{D}$, have the consequence that a gas element that enters through the shock front spends more time in the heating region before flowing down to settle onto the protoneutron star. Since it spends more time in the heating region, it can absorb more neutrino energy, increasing the overall efficiency of the neutrino mechanism.

The second reason has to do with turbulence and has become apparent only in the past few years. Turbulence is often analyzed employing Reynolds decomposition, a method that separates background flow from turbulent fluctuations. Using this method, one can show that turbulent fluctuations lead to an effective dynamical ram pressure (Reynolds stress) that contributes to the overall momentum balance between behind and in front of the stalled shock. The turbulent pressure is available only in 2D/3D simulations and it has been demonstrated (see, e.g., [23]) that because of this pressure, 2D/3D core-collapse supernovae explode with less thermal pressure, and, consequently with less neutrino heating.

Now, the Reynolds stress is dominated by turbulent fluctuations at the largest physical scales: A simulation that has more kinetic energy in large-scale motions will explode more easily than a simulation that has less. This realization readily explains recent findings by multiple simulation groups: $2 \mathrm{D}$ simulations appear to explode more readily than 3D simulations [22], [23]. This is likely a consequence of the different behaviors of turbulence in 2D and 3D. In 2D, turbulence transports kinetic energy to large scales (which is unphysical), artificially increasing the turbulent pressure contribution. In 3D, turbulence cascades energy to small scales (as it should and is known experimentally), so a 3D supernova will generally have less turbulent pressure support than a 2D supernova.

Another recent finding by multiple groups is that simulations with lower spatial resolution appear to explode more readily than simulations with higher resolution. There are two possible explanations for this and it is likely that they play hand-in-hand: (1) Low resolution creates a numerical bottleneck in the turbulent cascade, artificially trapping turbulent kinetic energy at large scales where it can contribute most to the explosion. (2) Low resolution also increases the size of numerical perturbations that enter through the shock and from which buoyant eddies form. The larger these seed perturbations are, the stronger is the turbulent convection and the larger is the Reynolds stress.

The qualitative and quantitative behavior of turbulent flow is very sensitive to numerical resolution. This can be appreciated by looking at Figure 7 which shows the same 3D simulation of neutrino-driven convection at 4 different resolutions, spanning a factor of 12 from the reference resolution that is presently used in many 3D simulations and which underresolves the turbulent flow. As resolution is increased, turbulent flow breaks down to progressively smaller features. What also occurs, but cannot be appreciated from a still figure, is that the intermittency of the flow increases as the turbulence is better resolved. This means that flow features are not persistent, but quickly appear and disappear through non-linear interactions of turbulent eddies. In this way, the turbulent cascade can be temporarily reversed (this is called backscatter in turbulence jargon), creating large-scale intermittent flow features similar to what is seen at low resolution. The role of intermittency in neutrino-driven turbulence and its effect on the explosion 


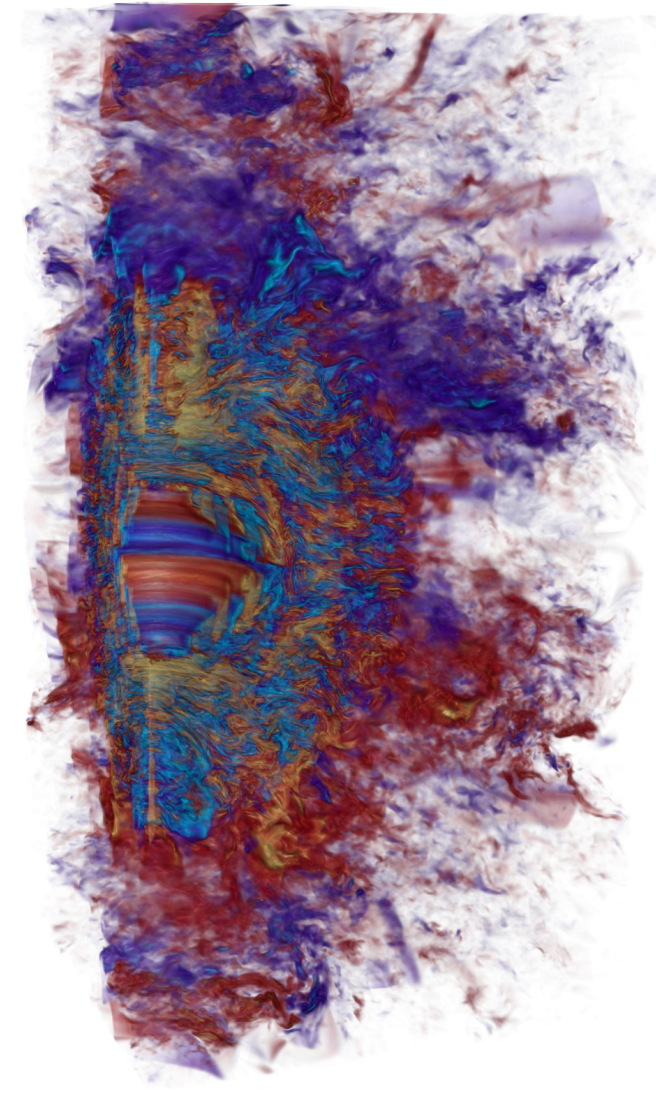

Fig. 8. Visualization of the toroidal magnetic field built up by an inverse cascade (large-scale dynamo) from small-scale magnetoturbulence in a magnetorotational core-collapse supernova. Shown is a $140 \times 70 \mathrm{~km} \mathrm{3D}$ octant region with periodic boundaries on the $x-z$ and $y-z$ faces. Regions of strongest positive and negative magnetic field are marked by light blue and yellowish colors. Dark blue and dark red colors mark regions of weake negative and positive magnetic field. Based on the NSF/NCSA Blue Waters simulations of Mösta et al. 2015 [24] and rendered by Robert R. Sisneros (NCSA) and Philipp Mösta (UC Berkeley).

mechanism remain to be studied.

A key challenge for 3D core-collapse supernova simulations is to provide sufficient resolution so that kinetic energy cascades away from the largest scales at the right rate. Resolution studies suggests that this may require between twice to ten times the resolution of current 3D simulations [21]. A ten-fold increase in resolution in 3D corresponds to a 10,000 times increase in the computational cost. An alternative may be to devise an efficient sub-grid model that, if included, provides for the correct rate of energy transfer to small scales. Work in that direction is still in its infancy in the core-collapse supernova context.

\section{MaKing Magnetars:}

\section{RESOLVING THE MAGNETOROTATIONAL INSTABILITY}

The magnetorotational mechanism relies on the presence of an ultra-strong $\left(\sim 10^{15}-10^{16} \mathrm{G}\right)$ global, primarily toroidal, magnetic field around the protoneutron star. Such a strongly magnetized protoneutron star is called a protomagnetar.

It has been theorized that the magnetorotational instability (MRI, [7]) could generate a strong local magnetic field that could be transformed into a global field by a dynamo process. While appealing, it was not at all clear that this is what happens. The physics is fundamentally global and 3D and global 3D MHD simulations with sufficient resolution to capture MRI-driven field growth were impossible to perform for core-collapse supernovae.

This changed with the advent of Blue Waters-class petascale supercomputers and is a testament to how increased compute power and capability systems like Blue Waters facilitate scientific discovery. In Mösta et al. 2015 [24], our group at Caltech carried out full-physics 3D global general-relativistic MHD simulations of ten milliseconds of a rapidly spinning protoneutron star's life, starting shortly after core bounce. We cut out a central octant (with appropriate boundary conditions) from another, lower-resolution 3D AMR simulation, and covered a $3 \mathrm{D}$ region of $140 \times 70 \times 70 \mathrm{~km}$ with uniform resolution. We performed four simulations to study the MHD dynamics at resolutions of $500 \mathrm{~m}$ ( $\sim 2$ points per MRI wavelength), $200 \mathrm{~m}$, $100 \mathrm{~m}$, and $50 \mathrm{~m}$ ( $\sim 20$ points per MRI wavelength). Since we employed uniform resolution and no AMR, the simulations showed excellent strong scaling. The $50 \mathrm{~m}$ simulation was run on 130, 000 Blue Waters cores. It consumed roughly 3 million Blue Waters node hours ( 48 million CPU hours).

Our simulations with $100 \mathrm{~m}$ and $50 \mathrm{~m}$ resolution resolve the MRI and show exponential growth of the magnetic field. This growth saturates at small scales within a few milliseconds and is consistent with what one anticipates on the basis of analytical estimates. The MRI drives MHD turbulence that is most prominent in the layer of greatest rotational shear, just outside of the protoneutron star core at radii of $20-30 \mathrm{~km}$. What we did not anticipate is that in the highest-resolution simulation (which resolves the turbulence best), an inverse turbulent cascade develops that transports magnetic field energy toward large scales. It acts as a large-scale dynamo that builds up global, primarily toroidal field, just in the way needed to power a magnetorotational explosion. Figure 8 shows the final toroidal magnetic field component in our $50 \mathrm{~m}$ simulation after $10 \mathrm{~ms}$ of evolution time. Regions of strongest positive and negative magnetic field are marked by yellowish and light blue colors, respectively, and are just outside the protoneutron star core. At the time shown, the magnetic field on large scales has not yet reached its saturated state. We expect this to occur after $\sim 50 \mathrm{~ms}$, which could not be simulated.

The results of Mösta et al. suggest that the conditions necessary for the magnetorotational mechanism are a generic outcome of the collapse of rapidly rotating cores. The MRI is a weak field instability and will grow to the needed saturation field strengths from any small seed magnetic field. The next step is to find a way to simulate for longer physical time and with a larger physical domain. This will be necessary in order to determine the long-term dynamical impact of the generated large-scale magnetic field. Such simulations will require algorithmic changes to improve parallel scaling, facilitate the efficient use of accelerators, and may require even larger and faster machines than Blue Waters. 


\section{CONCLUDING REMARKS}

Core-collapse supernova theorists have always been among the top group of users of supercomputers. The CDCs and IBMs of the 1960s and 1970s, the vector Crays of the 1970s to 1990s, the large parallel scalar architectures of the 2000s, and the current massively parallel SIMD machines all paved the path of progress for core-collapse supernova simulations.

Today's 3D simulations are rapidly improving in their included macroscopic and microscopic physics. They are beginning to answer decades-old questions and are allowing us to formulate new questions. There is still much need for improvement, which will come at no small price in the postMoore's-law era of heterogeneous supercomputers.

One important issue that the community must address is the reproducibility of simulations and the verification of simulation codes. It still occurs more often than not that different codes starting from the same initial conditions and implementing nominally the same physics arrive at quantitatively and qualitatively different outcomes. In the mid-2000s an extensive comparison of 1D supernova codes took place that provided results that are still being used as benchmarks today [13]. Efforts are now underway that will lead to the definition of multi-D benchmarks. In addition to code comparisons, the increasing availability of open-source simulation codes and routines for generating input physics (e.g., neutrino interactions) is furthering reproducibility. Importantly, these open-source codes now allow new researchers to enter the field without the need of spending many years on developing basic simulation technology that already exists.

Core collapse is, in essence, an initial value problem. Current simulations, even those in 3D, start from spherically symmetric precollapse conditions from 1D stellar evolution codes. However, stars rotate and convection in the layers surrounding the inert iron care is violently aspherical. These asphericities have an impact on the explosion mechanism. In order for 3D core-collapse supernova simulations to provide robust and reliable results, the initial conditions must be reliable and robust, and will likely require simulating the final phases of stellar evolution in 3D [20], which is another multi$\mathrm{D}$, multi-scale, multi-physics problem.

Neutrino quantum-kinetics for including neutrino oscillations directly into simulations will be an important, but exceedingly algorithmically and computationally challenging addition to the simulation physics. Formalisms for doing so are under development and first implementations (in spatially 1D) simulations may be available in a few years.

A single current top-of-the-line 3D neutrino radiationhydrodynamics simulation can be carried out to $\sim 0.5-$ 1 second after core bounce at a cost of several tens of millions of CPU hours; and it still underresolves the neutrino-driven turbulence. What is needed now, are many such simulations for studying sensitivity to initial conditions such as rotation and progenitor structure and input physics. These simulations should be at higher resolution and carried out for longer so that the longer-term development of the explosion (or collapse to a black hole) and, for example, neutron star birth kicks can be reliably simulated.
Many longer simulations at higher resolution will require much more compute power than is currently available. The good news is that the next generation of petascale systems and, certainly, exascale machines in the next decade will provide the necessary FLOPS. The bad news: the radical and disruptive architectural changes necessary on the route to exascale will require equally disruptive changes in supernova simulation codes. Already at petascale, the traditional data-parallel, linear/sequential execution model of all present supernova codes is the key limiting factor of code performance and scaling. A central issue is the need to communicate many boundary points between subdomains for commonly employed high-order finite difference and finite volume schemes. With increasing parallel process count, communication eventually dominates over computation in current supernova simulations.

Since latencies cannot be hidden, efficiently offloading data and tasks to accelerators in heterogeneous systems is difficult for current supernova codes. The upcoming generation of petascale machines such as DOE's Summit and Sierra, fully embraces heterogeneity. For exascale machines, power consumption will be the driver of computing architecture. Current Blue Waters already draws $\sim 10 \mathrm{MW}$ of power and there is not much upwards flexibility for future machines. Unless there are unforeseen breakthroughs in semiconductor technology that provide increased single-core performance at orders of magnitude lower power footprint, exascale machines will likely be all-accelerator with hundreds of millions of slow, highly energy efficient cores.

Accessing the compute power of upcoming petascale and future exascale machines requires a radical departure from current code design and major code development efforts. Several supernova groups are exploring new algorithms, numerical methods, and parallelization paradigms. Discontinuous Galerkin (DG) finite elements (e.g., [25]) have emerged as a promising discretization approach that guarantees high numerical order while minimizing the amount of subdomain boundary information that needs to be communicated between processes. In addition, switching to a new, more flexible parallelization will likely be necessary to prepare supernova codes (and other computational astrophysics codes solving similar equations) for exascale machines. A prime contender being considered by supernova groups is task-based parallelism, which allows for fine-grained dynamical load balancing and asynchronous execution and communication. Frameworks that can become task-based backbones of future supernova codes already exist, e.g., Charm++ (http://charm. cs.illinois.edu/research/charm), Legion (http://legion.stanford. edu/overview/), and Uintah (http://uintah.utah.edu/).

\section{ACKNOWLEDGMENTS}

I acknowledge helpful conversations with and help from Adam Burrows, Sean Couch, Steve Drasco, Roland Haas, Kenta Kiuchi, Philipp Mösta, David Radice, Luke Roberts, Erik Schnetter, Ed Seidel, and Masaru Shibata. I thank the Yukawa Institute for Theoretical Physics at Kyoto University for hospitality while writing this article. This work is supported by NSF under award nos. CAREER PHY-1151197 
and TCAN AST-1333520, and by the Sherman Fairchild Foundation. Computations were performed on NSF XSEDE under allocation TG-PHY100033 and on NSF/NCSA BlueWaters under NSF PRAC award no. ACI-1440083. Movies of simulation results can be found on http://www.youtube.com/ SXSCollaboration

\section{REFERENCES}

[1] C. D. Ott, E. Abdikamalov, P. Mösta, R. Haas, S. Drasco, E. P. O'Connor, C. Reisswig, C. A. Meakin, and E. Schnetter, "Generalrelativistic Simulations of Three-dimensional Core-collapse Supernovae," Astrophys. J., vol. 768, 115, May 2013.

[2] H. A. Bethe and J. R. Wilson, "Revival of a stalled supernova shock by neutrino heating," Astrophys. J., vol. 295, 14, Aug. 1985.

[3] H.-T. Janka, "Explosion Mechanisms of Core-Collapse Supernovae," Ann. Rev. Nuc. Par. Sci., vol. 62, 407, Nov. 2012.

[4] P. Mösta, S. Richers, C. D. Ott, R. Haas, A. L. Piro, K. Boydstun, E. Abdikamalov, C. Reisswig, and E. Schnetter, "Magnetorotational Core-Collapse Supernovae in Three Dimensions," Astrophys. J. Lett., vol. 785, L29, Apr. 2014.

[5] G. S. Bisnovatyi-Kogan, "The Explosion of a Rotating Star As a Supernova Mechanism.” Astron. Zh., vol. 47, 813, Aug. 1970.

[6] J. M. LeBlanc and J. R. Wilson, "A Numerical Example of the Collapse of a Rotating Magnetized Star," Astrophys. J., vol. 161, 541, Aug. 1970.

[7] S. A. Balbus and J. F. Hawley, "A powerful local shear instability in weakly magnetized disks. I-Linear analysis. II-Nonlinear evolution," Astrophys. J., vol. 376, 214, Jul. 1991.

[8] A. Wongwathanarat, H. Janka, and E. Müller, "Hydrodynamical Neutron Star Kicks in Three Dimensions," Astrophys. J. Lett., vol. 725, L106, Dec. 2010.

[9] C. D. Ott, A. Burrows, L. Dessart, and E. Livne, "Two-Dimensional Multiangle, Multigroup Neutrino Radiation-Hydrodynamic Simulations of Postbounce Supernova Cores," Astrophys. J., vol. 685, 1069, 2008.

[10] E. F. Toro, Riemann Solvers and Numerical Methods for Fluid Dynamics. Berlin: Springer, 1999.

[11] T. W. Baumgarte and S. L. Shapiro, Numerical Relativity: Solving Einstein's Equations on the Computer. Cambridge, UK: Cambridge University Press, 2010.

[12] T. Kuroda, T. Takiwaki, and K. Kotake, "A New Multi-energy Neutrino Radiation-Hydrodynamics Code in Full General Relativity and Its Application to the Gravitational Collapse of Massive Stars," Astrophys. J. Supp. Ser. , vol. 222, 20, Feb. 2016.

[13] M. Liebendörfer, M. Rampp, H.-T. Janka, and A. Mezzacappa, "Supernova Simulations with Boltzmann Neutrino Transport: A Comparison of Methods," Astrophys. J., vol. 620, 840, Feb. 2005.

[14] K. Sumiyoshi, T. Takiwaki, H. Matsufuru, and S. Yamada, "Multidimensional Features of Neutrino Transfer in Core-collapse Supernovae," Astrophys. J. Supp. Ser. , vol. 216, 5, Jan. 2015.

[15] E. O'Connor and S. M. Couch, "Two Dimensional Core-Collapse Supernova Explosions Aided by General Relativity with Multidimensional Neutrino Transport," submitted to Astrophys. J.; arXiv:1511.07443. Nov. 2015.

[16] L. F. Roberts, C. D. Ott, R. Haas, E. P. O'Connor, P. Diener, and E. Schnetter, "General Relativistic Three-Dimensional Multi-Group Neutrino Radiation-Hydrodynamics Simulations of Core-Collapse Supernovae," To appear in Astrophys. J.; arXiv:1604.07848 Apr. 2016.

[17] A. Mirizzi, I. Tamborra, H.-T. Janka, N. Saviano, K. Scholberg, R. Bollig, L. Hüdepohl, and S. Chakraborty, "Supernova neutrinos: production, oscillations and detection," Nuovo Cimento Rivista Serie, vol. 39, pp. 1112, 2016.

[18] A. Vlasenko, G. M. Fuller, and V. Cirigliano, "Neutrino quantum kinetics," Phys. Rev. D., vol. 89, no. 10, 105004, May 2014.

[19] A. W. Steiner, M. Hempel, and T. Fischer, "Core-collapse Supernova Equations of State Based on Neutron Star Observations," Astrophys. J., vol. 774, 17, Sep. 2013.

[20] S. M. Couch, E. Chatzopoulos, W. D. Arnett, and F. X. Timmes, "The Three-dimensional Evolution to Core Collapse of a Massive Star," Astrophys. J. Lett., vol. 808, L21, Jul. 2015.

[21] D. Radice, C. D. Ott, E. Abdikamalov, S. M. Couch, R. Haas, and E. Schnetter, "Neutrino-driven Convection in Core-collapse Supernovae: High-resolution Simulations," Astrophys. J., vol. 820, 76, Mar. 2016.
[22] E. J. Lentz, S. W. Bruenn, W. R. Hix, A. Mezzacappa, O. E. B. Messer, E. Endeve, J. M. Blondin, J. A. Harris, P. Marronetti, and K. N. Yakunin, "Three-dimensional Core-collapse Supernova Simulated Using a $15 \mathrm{M}_{\odot}$ Progenitor," Astrophys. J. Lett., vol. 807, L31, Jul. 2015.

[23] S. M. Couch and C. D. Ott, "The Role of Turbulence in Neutrinodriven Core-collapse Supernova Explosions," Astrophys. J., vol. 799, 5, Jan. 2015.

[24] P. Mösta, C. D. Ott, D. Radice, L. F. Roberts, R. Haas, and E. Schnetter, "A Large-Scale Dynamo and Magnetoturbulence in Rapidly Rotating Core-Collapse Supernovae," Nature, vol. 528, no. 7582, pp. 376-379, Dec. 2015.

[25] J. S. Hesthaven and T. Warburton, Nodal Discontinuous Galerkin Methods: Algorithms, Analysis, and Applications, 1st ed. Springer, 2007. 